International Journal of Modern Physics D

(C) World Scientific Publishing Company

\title{
Laboratory Constraints
}

Philippe Brax, Clare Burrage and Anne-Christine Davis

Received Day Month Year

Revised Day Month Year

\section{Contents}

1. Introduction . . . . . . . . . . . . . . . . . . . . . . . . . . . .

2. Experimental Constraints . . . . . . . . . . . . . . . . . 3

2.1. Chameleons in Laboratory Vacuums . . . . . . . . . . . . . . . . . . 3

2.2. Unscreening Inside the Vacuum Chamber . . . . . . . . . . . . . . . . 4

2.3. A comparison with astrophysical tests . . . . . . . . . . . . . . . . . 5

2.4. Atom Interferometry . . . . . . . . . . . . . . . . . . . . . . . . . . . . . . . . . .

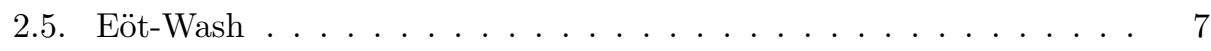

2.6. Casimir . . . . . . . . . . . . . . . . . . . 7

2.7. Quantum Bouncing Neutrons . . . . . . . . . . . . . . . . 8

2.8. Precision Atomic Tests . . . . . . . . . . . . . . . . . . . . . . . . 10

3. The Symmetron . . . . . . . . . . . . . . . . . . . . . . . . . . . . . . . . . . . . . . .

4. The Field Profile in a Cylinder . . . . . . . . . . . . . . . . . . . . . . . . 11

5. The Dilaton as a Worked out Example . . . . . . . . . . . . . . . . . . . . 14

5.1. The model . . . . . . . . . . . . . . . . . . . 14

$5.2 . \quad$ Laboratory experiments . . . . . . . . . . . . . . . . 16

6. Tomographic parameterisation . . . . . . . . . . . . . . . . 17

7. Vainshtein mechanism . . . . . . . . . . . . . . . . . . 20

8. Coupling to Photons . . . . . . . . . . . . . . . . . . . . . . . . . . . . . . . . . . . . 22

8.1. Quantum Coupling . . . . . . . . . . . . . . . . . . . . 23

8.2. Photon-scalar mixing . . . . . . . . . . . . . . . . . . 23

8.3. Scalar reflection . . . . . . . . . . . . . . . . . . . 25

8.4. Experimental results . . . . . . . . . . . . . . . . 26

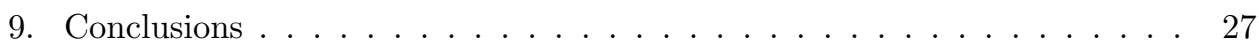

\section{Introduction}

Dark energy is a cosmological phenomenon per se. In this chapter we will describe attempts to detect effects of the physics of modified gravity, motivated by dark energy and the cosmological constant problem, in the laboratory. Classical effects of modified gravity can be tested by fifth force searches where new classical interactions could influence the motion of test masses. The quantum nature of the modifications 
can also be probed using the Casimir effect, atom and neutron interferometry, and the neutron energy levels in vacuum. Theoretically we have restricted the models to scalar-tensor theories with a coupling between a scalar field and matter. The coupling to photons and its quantum-mechanical origin is also recalled.

Laboratory tests of gravity have a long history and, as discussed in Section ??, the need to make cosmological theories of dark energy and modified gravity compatible with laboratory and solar system tests was a key motivation for the introduction of screening mechanisms. In broad terms the goal of screening mechanisms is to allow the additional scalar field to give rise to modifications of the standard cosmology on the largest scales in the universe, whilst being un-observable on the shorter distances and at the higher densities present on Earth. However this is not the end of the story, as we will see in this section; carefully designed experiments can allow the effects of the scalar field to be unscreened. The additional level of precision and control that we have in the laboratory then means that these measurements tend to be extremely constraining for theories of dark energy, and cosmological modified gravity theories.

In the last ten years, the variety of experimental techniques which have been introduced to test dark energy is quite astonishing. From the classic Casimir and fifth force experiments to levitating microspheres and atomic interferometry, the effects of the scalar fields are all in the non-linear regime of the theories. This is different from most of the tests on cosmological scales where the linear regime is the easiest to probe. Hence the laboratory experiments are a useful complement to future large cosmological surveys. On the other hand, and as the non-linear regime is what will be the subject of this review chapter, the analysis has to be mostly dealt with in a case by case basis. No model independent parameterised description is yet available for laboratory tests, and the non-linear regime they probe can only be connected to the parameterised descriptions of the linear perturbation theory relevant on the largest cosmological scales on a case by case basis. As there are already similar reviews in the literature Joyce et al.(2015)Joyce, Jain, Khoury, and Trodden Bull et al.(2016) Burrage and Sakstein(2016) Burrage and Sakstein(2017) we have decided to concentrate some of the technical aspects to less developed models such as the environmentally dependent dilaton which can be treated almost completely analytically and provides a nice template for more complex models.

The types of laboratory tests that will be presented here really only probe the screening properties of dark energy theories, i.e. the fact that very light scalar fields involved in the late time acceleration of the expansion rate of the Universe would induce far too large deviations from General Relativity in the solar system and therefore must be shielded from matter locally. Types of screening can be classified in two different ways: Firstly by the highest order of the derivative terms which appear in the non-linear terms. From zero to two derivatives these are: chameleon, K-mouflage and Vainshtein. Secondly by the class of term in the scalar Lagrangian in which the non-linear terms are present. For the chameleon, symmetron (or dilaton) and kinetic-chameleon models (Vainshtein and Galileon) the non-linearities appear 
in the scalar potential, matter coupling and kinetic terms respectively. Unfortunately, so far, it does not appear to be possible to probe screening which relies on derivative self interactions with laboratory experiments. This is because the nature of the screening means that variations in the field occur only over longer distance scales than can be probed terrestrially. Only chameleon and symmetron or dilaton models are sufficiently local to respond to variations of matter densities on laboratory scales. They will be the main focus of this review although the techniques presented here can be applied to other models too.

\section{Experimental Constraints}

Laboratory experiments are most effective at constraining theories which screen through a chameleon-like mechanism. Chameleon theories have the advantage that the scalar field responds rapidly to changes in density, meaning that even if the effects of the scalar are screened in the solar system they can be unscreened by a laboratory vacuum chamber. In this section we will first describe how a chameleon scalar behaves in a laboratory vacuum, and then go on to detail the laboratory experiments which currently are the most constraining for chameleon models. We will discuss in the following section the case of the dilaton which corresponds to the Damour-Polyakov screening, and we will see that laboratory experiments are less effective.

\subsection{Chameleons in Laboratory Vacuums}

The chameleon scalar field changes its mass as a function of the local density. We consider here an idealized vacuum chamber, which is spherical with internal radius $L$, internal density $\rho_{\text {vac }}$ and walls of density $\rho_{\text {wall }}$ and thickness $T$. If $T>1 / m\left(\rho_{\text {wall }}\right)$ then we know that within the walls of the vacuum chamber the chameleon reaches the field value which minimises its effective potential. This greatly simplifies our calculations, as it means that we can ignore the behaviour of the chameleon in the exterior of the vacuum chamber, and just focus on the interior, as long as we impose the boundary condition that the chameleon minimises its effective potential within the walls. Whilst the condition $T>1 / m\left(\rho_{\text {wall }}\right)$, needs to be checked experiment by experiment, and chameleon model by chameleon model, in general we find that this is satisfied for chameleon models of interest if $T \gtrsim 1 \mathrm{~mm}$.

In the interior of the vacuum chamber the density is much less than within the walls, therefore the chameleon will try to adjust its value to reach the value which minimises the effective potential for this lower density. If $L>$ $1 / m\left(\rho_{\mathrm{vac}}\right)$ then at the center of the vacuum chamber the chameleon will have reached this minimising value. For smaller vacuum chambers the chameleon field will still evolve in the interior, it just will not have enough room to reach the value which minimises the effective potential. In this case we find that at the centre of the vacuum chamber the chameleon takes a value so that its Compton wavelength is of order the size of the vacuum chamber 
$1 / m\left(\rho_{\text {vac }}\right) \sim L$. The order one constant of proportionality varies depending on the choice of chameleon model Burrage and Sakstein(2016) Burrage and Sakstein(2017) It is not generally possible to solve analytically for the full form of the chameleon profile inside the vacuum chamber, but it is possible to compute it numerically Elder et al.(2016)Elder, Khoury, Haslinger, Jaffe, Mller, and Hamilton, Schlgel et al.(2016)Schlgel, Clesse, and Fzfa

\subsection{Unscreening Inside the Vacuum Chamber}

The conditions for a source mass to be screened are given in Equation (??) [reference to early chapter] and will be reviewed in the next section in Equation (346). Whether or not an object is screened depends on the value of the scalar field in the interior of the source mass, and the background value that the scalar would take if the source were absent. The advantage of performing experiments in laboratory vacuua is that, if the field is able to respond to the lower density of the vacuum as described in the previous subsection, then the difference between the background value of the scalar field, and the value that minimises the potential in the interior is increased, and the conditions for screening are satisfied for fewer objects.

In particular it has been shown that, at least in parts of the chameleon parameter space neutrons, atomic nuclei and silica microspheres can be unscreened in vacuua with $L \sim 10 \mathrm{~cm}$ and $\rho_{\text {vac }} \sim 10^{-17} \mathrm{~g} / \mathrm{cm}^{3}$.

In the following sub-sections we review the most constraining experiments for chameleon models. These constraints are summarized in Figure 1 .

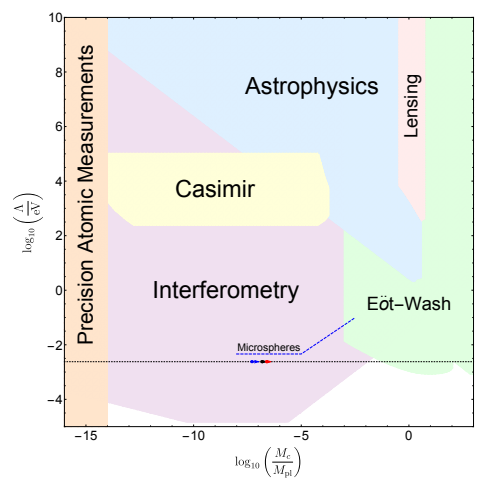

Fig. 1. Experimental constraints on the chameleon self-coupling $\Lambda$ and coupling to matter $M_{c}=$ $\frac{M_{\mathrm{PI}}}{\beta}$, for the chameleon potential $V(\phi)=\Lambda^{5} / \phi$. 


\subsection{A comparison with astrophysical tests}

In the astrophysical tests of screened and unscreened models of dark energy and modified gravity it is often useful to use

$$
\chi_{\mathrm{BG}}=\frac{\phi_{\mathrm{BG}}}{2 \beta_{\mathrm{BG}} m_{\mathrm{Pl}}}
$$

in order to discriminate screened and unscreened objects. Here $B G$ refers to the background in which a given object is embedded, where $\phi_{\mathrm{BG}}$ and $\beta_{\mathrm{BG}}$ refer to the background value of the scalar field and the background coupling to matter. A given object is screened in this environment when

$$
\Phi_{A} \geqslant \chi_{\mathrm{BG}}
$$

where $\Phi_{A}$ is the Newtonian potential at the surface of the object $A$. This is particularly useful in astrophysics where a host of phenomena happen in the same environment, e.g. within a galaxy where for instance one may be interested in the screening of stars or the galactic gas. Hence a uniform criterion depending uniquely on the Newtonian potential makes sense and is practical. For laboratory experiments, the background is far from being standardisable, i.e. it could be a cylindrical cavity, the two plates of Casimir experiments or the single mirror for neutron energy levels. As a result it is far more efficient to introduce the scalar charge

$$
Q_{A}=\frac{\left|\phi_{\mathrm{BG}}-\phi_{c}\right|}{2 m_{\mathrm{Pl}} \Phi_{A}}
$$

where $\phi_{c}$ is the value of the scalar at the center of the source object. For a specific object in a given environment Newton's law is corrected as

$$
V_{A B}=-\frac{m_{A} m_{B}}{r}\left(1+2 Q_{A} Q_{B}\right)
$$

when the interaction range of the scalar is much smaller than the distances probed by the experiment, and $V_{A B}$ is the interaction potential between two masses. The two bodies are screened if

$$
Q_{A, B} \leqslant \beta_{\mathrm{BG}}
$$

and when screening is not operating $Q_{A, B} \rightarrow \beta_{\mathrm{BG}}$ in $V_{A B}$. This is what will be used in the following. For instance for atomic interferometry, the atoms will be unscreened whilst the source object will be. The ratio of the charge of the object to the background coupling can be identified with $3 \frac{\delta R}{R}=\frac{\delta M}{M}$ where $\delta R$ is the size of the thin shell over which the field varies inside the object and creates the interaction with another object. Deep inside the object the field is constant as the mass of the scalar field is too large to allow for any propagation of the scalar from the inside to the outside of the object. When bodies are not spherical, or nearly spherical, as for the Casimir effect where infinite parallel plates are used, screening operates when

$$
m_{\text {plate }} d \gg 1
$$


where $m_{\text {plate }}$ is the mass of the chameleons inside the plates and $d$ their width. For most models this implies that the field effectively vanishes inside the plates and varies between the plates where near vacuum has been realised.

\subsection{Atom Interferometry}

Atom interferometry relies on the wave-particle duality of atoms. The wave function of an atom is split into two parts, which describe the center of mass position traveling along two different paths. The paths start and end at the same point, but are spatially separated otherwise. At the final point the phases of the two parts of the wavefunction interfere, allowing for a difference in the phase accumulated along the two paths to be detected (somewhat analogously to the double slit experiment).

The spatial position of the atoms within the experiment can be controlled using lasers. Once they are cooled sufficiently, the momentum of an atom can be increased by absorption of a laser photon with frequency tuned to a particular atomic excitation, as conservation of momentum requires the atom to inherit the three momentum of the absorbed photon. Similarly the atom can be made to lose momentum by stimulated emission. The probability of whether the atoms absorb (or emit) a photon can also be controlled. So that with three laser pulses it is possible to split the atomic wavefunction into two parts (the analogue of the beam splitter in a classical interferometer) to reverse the direction of motion of the atoms (the analogue of the mirrors) and to recombine the wavefunction (the analogue of the second beam splitter).

Differences in the phase of the wavefunction along the two paths can result either from a difference in the accumulated action along each path, or from differences in the phase inherited from the photon at each interaction. If the atoms are experiencing a constant acceleration along the direction described by the laser pulses, then the difference in phase is particularly simple. As moving the atoms around within the experiment directly correlates with whether the atoms are in the excited or unexcited state it is most convenient to express this in terms of the probability of finding the atom in the excited state at the output of the interferometer.

$$
P \propto \cos ^{2}\left[\frac{a k T^{2}}{\hbar}\right],
$$

where $k$ is the photon momentum, $T$ is the time between laser pulses (so that $2 T$ is the duration of the experiment), and $a$ is the constant acceleration.

An acceleration $a$ can be caused by the gravitational, or chameleon forces due to a massive source object being placed inside the vacuum chamber. This massive source is typically sufficiently large that it will be screened, but the atomic nuclei are small enough that, as discussed in the previous subsection, they are unscreened over a large range of the parameter space Burrage et al.(2015)Burrage, Copeland, and Hinds Burrage and Copeland(2016)|Elder et al.(2016)Elder, Khoury, Haslinger, Jaffe, making them sensitive probes of the chameleon field. Experiments searching for chameleon accelerations with atom interferometry have reached a sensitivity of 
$10^{-8} \mathrm{~g}\left(\mathrm{~g} \equiv G M_{\oplus} / R_{\oplus}\right.$ is the gravitational acceleration at the surface of the Earth) Hamilton et al.(2015)Hamilton, Jaffe, Haslinger, Simmons, Mller, and Khoury Burrage et al.(2016)Burrage, Kuribayashi-Colema

\subsection{Eöt-Wash}

Torsion balance experiments have a long history of searching for fifth forces, and modifications of gravity. The principle underlying the experiments is to have one or more test masses suspended, and to look for deflections of the test masses towards source masses by measuring the torsion in the suspension of the test masses. Commonly the source and test masses are arranged so that the inverse-square contribution to the total force is canceled, and the experiment is sensitive to deviations from standard gravity.

The current best constraints come from the Eöt-Wash experiment, Adelberger et al.(2003)Adelberger, Heckel, and Nelson Kapner et al.(2007)Kapner, Cook, Adelberger, Gundlach, Heckel, Hoyle, an which uses circular disks for the masses. The disks have holes bored in them, and are arranged one above the other so that if there are no modifications to gravity there is expected to be no net torque of one plate rotating to bring the massive parts of the disk close to the more massive parts of the second disk.

One of the challenges of these experiments is to reduce as far as possible any electromagnetic forces between the plates that could be mistaken for modifications of gravity. One of the ways that this is done in the Eöt-Wash experiment is to place a beryllium-copper membrane between the plates. This still allows the experiment to search for fifth forces that are not screened, but the presence of the plate can act to screen out, for example, chameleon forces between the plates Brax et al.(2008)Brax, van de Bruck, Davis, and Shaw Adelberger et al.(2007)Adelberger, Heckel, Hoedl, Hoyle, Kapner, and Up This reduces the sensitivity of the experiment to screened fifth forces.

\subsection{Casimir}

The Casimir force is an effect predicted by quantum electrodynamics, which is absent in classical physics. It is the force that arises between two parallel plates, placed in vacuum, due to the quantum fluctuations of the electromagnetic field in the space between the plates. This force scales as $d^{-4}$, where $d$ is the distance between the plates, and therefore is most easily detected when the plates are placed close together, current experiments probe sub-mm and sub-micron distance scales Lamoreaux and Buttler(2005) Lambrecht and Reynaud(2011)

If fifth forces exist they could also be detected by an experiment searching for Casimir effects. These experiments are particularly sensitive to screening through the thin-shell effect [we need to check that thinshell is defined in an earlier chapter], as close to the surface of a source the field is changing rapidly, giving rise to potentially detectable forces. The chameleon force (per unit area) between two plates scales 
as Mota and Shaw(2007) Brax et al.(2007a)Brax, van de Bruck, Davis, Mota, and Shaw Brax and Davis(2015)

$$
\frac{F_{\text {cham }}}{A} \propto d^{-\frac{2 n}{n+2}},
$$

The experimental challenge for such a search, is to make the two plates perfectly smooth and to keep the plates perfectly parallel. In practice it may be easier to search for the Casimir effect between a plate and a sphere, or between two spheres instead. In this case, the Casimir force scales as $d^{-3}$ and the chameleon force would scale as

$$
\frac{F_{\text {cham }}}{A} \propto d^{\frac{2-n}{n+2}} .
$$

Current searches for the Casimir force are most constraining for chameleon models with $n=-4$ and $n=-6$ when $\Lambda_{c}$ is fixed to the dark energy scale. A new generation of these experiments, specifically tailored to look for the chameleon force with parallel plates and larger separations is currently being developed Lambrecht et al.(2005)Lambrecht, Nesvizhevsky, Onofrio, and Reynaud Lamoreaux and Buttler(2005)

Further sensitivity to
the chameleon could be obtained by varying the density of the gas between the two plates Brax et al.(2010a)Brax, van de Bruck, Davis, Shaw, and Iannuzzi Almasi et al.(2015)Almasi, Brax, Iannuzzi, and Sedmik

\subsection{Quantum Bouncing Neutrons}

\section{Neutrons}

can be used to test for the presence of new interaction with the $q$ BOUNCE experiment Abele et al.(2010)Abele, Jenke, Leeb, and Schmiedmayer Jenke et al.(2011)Jenke, Geltenbort, Lemmel, and Abele Jenke et al.(201

These experiments use ultra-cold neutrons in the terrestrial gravitational potential above a mirror with a large enough Fermi potential to reflect neutrons totally. As first obtained in Nesvizhevsky et al.(2002) the energy eigenstates are of the neutrons are discrete. The basic setup can be found in Jenke et al.(2011)Jenke, Geltenbort, Lemmel, and Abele where the energy resolution between the level is as low as $3 \times 10^{-15} \mathrm{eV}{ }^{?}$ Recently in? the transitions between the energy ground state $E_{1}=1.40672 \mathrm{peV}$ and the excited states $E_{3}=3.32144$ $\mathrm{peV}$ as well as $E_{4}=4.08321 \mathrm{peV}$ have been observed. This can be achieved as follows. First, the neutrons encounter a state selector for the ground state $|1\rangle$ having energy $E_{1}$. This combines a polished mirror at the bottom and a rough absorbing scatterer at the top separated by about $20 \mu \mathrm{m}$. Neutrons in excited states with a diffuse wave function are scattered out of the system. Then a horizontal mirror undergoes harmonic oscillations with a tunable frequency $\omega$, which drives the system into a coherent superposition of ground and excited states. Finally the neutrons go through a selector which is identical to the first one and acting as a ground state selector. When neutron are excited to higher levels than the ground state in the second region, nothing is transmitted and a dip in the transmission rate at a given frequency is observed. These dips allow one to measure the energy differences between the ground state and typically the first few levels, e.g. the third and fourth. 
The quantum-mechanical description of a neutron above a mirror in the gravitational potential is given by the Schrödinger equation Westphal et al.(2007)Westphal, Abele, Baessler, Nesvizhevsky, Petukhov, Protasov, and Voronin

$$
-\frac{\hbar^{2}}{2 m} \frac{\partial^{2} \psi_{n}(z)}{\partial z^{2}}+m g z \psi_{n}(z)=E_{n} \psi_{n}(z)
$$

with a characteristic length scale

$$
z_{0}=\sqrt[3]{\frac{\hbar^{2}}{2 m^{2} g}}=5.87 \mu \mathrm{m},
$$

and a typical energy scale $E_{0}=\left(\left(\hbar^{2} m g^{2}\right) / 2\right)^{1 / 3}$ which are given by the mass $m$ of the neutron and the acceleration of the earth $g$. Above the mirror the normalized wavefunctions for $z>0$ read

$$
\psi_{n}^{(0)}(z)=C_{n}^{(1)} \operatorname{Ai}\left(\frac{z-z_{n}}{z_{0}}\right)
$$

with normalisation

$$
C_{n}^{(1)}=\frac{1}{\sqrt{z_{0}} \operatorname{Ai}^{\prime}\left(-\frac{z_{n}}{z_{0}}\right)}
$$

and $z_{n}=\frac{E_{n}}{m g}$. Here $z_{n}$ is the n-th zero of the Airy function which characterises the energy levels of the neutrons. Outside this region the wavefunctions vanish as the neutron do not penetrate inside the mirror. The first few energy levels are given in Table 1. When a new interaction of the chameleon type is present, the potential is

\begin{tabular}{|c|c|}
\hline State & Energy $[\mathrm{peV}]$ \\
\hline \hline$|1\rangle$ & $E_{1}=1.40672$ \\
\hline$|2\rangle$ & $E_{2}=2.45951$ \\
\hline$|3\rangle$ & $E_{3}=3.32144$ \\
\hline$|4\rangle$ & $E_{4}=4.08321$ \\
\hline$|5\rangle$ & $E_{5}=4.77958$ \\
\hline$|6\rangle$ & $E_{6}=5.42846$ \\
\hline
\end{tabular}

shifted to

$$
V(z)=m g z+m(A(\phi(z))-1)
$$

where $\phi(z)$ is the profile of the scalar field above the mirror. This can be easily obtained for chameleons or symmetrons for instance. The perturbations to the n-th 
energy level is obtained as, where $\langle z \mid n\rangle=\psi_{n}^{(0)}(z)$,

$$
\delta E_{n}=m\langle n|(A-\phi(z)-1)| n\rangle
$$

to first order in perturbation theory. This has to be less than the precision of order $3 \times 10^{-15} \mathrm{eV}$ and leads to interesting constrained on dark energy models.

\subsection{Precision Atomic Tests}

As we have discussed above atomic nuclei can be unscreened in a laboratory vacuum. In the atom interferometry experiments discussed above the atoms were test particles probing the chameleon field due to a macroscopic source. But the nuclei can also be considered as the source of a chameleon field that is probed by the orbiting electrons. If the unscreened chameleon force is very strong then this could cause measurable perturbations to atomic energy levels, with an extra contribution to the electron Hamiltonian of the form

$$
\delta H=\frac{m_{e}}{M} \phi_{\mathrm{N}},
$$

where $\phi_{\mathrm{N}}$ is the chameleon field sourced by the nucleus.

The most precise measurements currently are of the structure of hydrogenic atoms. The shifts to the lowest energy levels due to a chameleon force $\operatorname{are}$ Brax and Burrage(2011)

$$
\begin{array}{r}
\Delta E_{1 \mathrm{~s}}=-\frac{Z m_{N} m_{e}}{4 \pi a_{0} M^{2}} \\
\Delta E_{2 \mathrm{~s}}=\Delta E_{2 \mathrm{p}}=-\frac{Z m_{N} m_{e}}{16 \pi a_{0} M^{2}},
\end{array}
$$

where $Z$ is the atomic number, $m_{N}$ is the nucleon mass, and $a_{0}$ is the Bohr radius. The potential coupling of the chameleons to photons, discussed in the next section, will break the degeneracy between the $2 \mathrm{~S}$ and $2 \mathrm{P}$ levels.

The best measured transition is currently the $1 \mathrm{~S}-2 \mathrm{~S}$ transition in atomic hydrogen, with a total uncertainty of $10^{-9} \mathrm{eV}$ (at $1 \sigma)$ Jaeckel and Roy(2010) Schwob et al.(1999)Schwob, Jozefowski, de Beauvoir, Hilico, Nez, Julien, Biraben, Acef, Zondy, and Clairon]

No signs of a deviation from standard electromagnetism have been found, and so the chameleon coupling must be constrained to be

$$
M \gtrsim 10 \mathrm{TeV} \text {. }
$$

\section{The Symmetron}

The constraints from all of the experiments detailed above have also been studied for the symmetron model. The symmetron is similar to the chameleon in that it has canonical kinetic terms, and its screening is through terms that are non-linear in the field. However the difference between the models is that the chameleon can screen because it varies its mass with the environment, and the symmetron because 
it varies the strength of its coupling to matter. This occurs because the symmetron has a spontaneous symmetry breaking potential, and couples to matter in such a way that regions of high density can restore the symmetry. The resulting effective potential is:

$$
V_{\text {eff }}(\phi)=\frac{1}{2}\left(-\mu^{2}+\frac{\rho}{M^{2}}\right) \phi^{2}+\frac{\lambda}{4 !} \phi^{4}
$$

where $\mu$ is the bare mass of the symmetron, $M$ the energy scale controlling strength of the coupling to matter, and $\lambda$ the dimensionless constant controlling the self interactions of the field. The form of the coupling to matter also means that the symmetron fifth force experienced by a test particle is $\vec{F}=\phi \vec{\nabla} \phi / M^{2}$. The consequence of this is that when the symmetry is restored in regions where $\rho>\mu^{2} M^{2}$ the fifth force is switched off. As can be seen from Equation 20 the mass of the symmetron in the symmetry broken phase is approximately $\mu$. Unlike the chameleon, therefore, the symmetron does not have the ability to adjust its mass in the low density environment of laboratory vacuum chamber. If the Compton wavelength of the symmetron is larger than the size of the vacuum chamber, $\mu L \ll 1$, the field is not able to vary within the chamber and so no fifth force can be present. Conversely, if the Compton wavelength of the symmetron is smaller than the distances probed in the experiment (for example the distance between test and source masses) then the fifth force will be exponentially suppressed by the Yukawa term $e^{-m d}$ where $m$ is the symmetron mass and $d$ the distance between two objects. This means that any experiment is only sensitive to symmetron models whose masses fall between these two limits. This can be seen directly by considering the form of the symmetron field profile around a spherical source of radius $R$ and constant density embedded in a lower density background.

$$
\phi=\phi_{\text {out }}-\frac{\left(\phi_{\text {out }}-\phi_{\text {in }}\right) R e^{m_{\text {out }}(R-r)}}{r}\left(\frac{m_{\text {in }} R-\tanh m_{\text {in }} R}{m_{\text {in }} R+R m_{\text {out }} \tanh m_{\text {in }} R}\right)
$$

Inside the source the field reaches a minimum value $\phi=\phi_{\text {in }}$, and the mass of the field is $m_{\mathrm{in}}$. Similarly in the background surrounding the source the field takes the value $\phi=\phi_{\text {out }}$ and has mass $m_{\text {out }}$. This profile is exponentially suppressed at distances larger than $1 / m_{\text {out }}$ away from the source. It is also suppressed when $\phi_{\text {out }}=\phi_{\text {in }}$. This occurs when the mass of the field $m_{\text {out }}$ is too large for the field to evolve within the vacuum chamber.

Constraints on the symmetron model have not been computed for all of the experiments described above. But those from atom-interferometry, and from the Eöt-Wash experiment are shown in Figure 2 with constraints coming from astrophysical observations included for comparison.

\section{The Field Profile in a Cylinder}

We have mentioned several times already that the chameleon field can "resonate" inside cavities. This can be made completely explicit using a simple model of a 


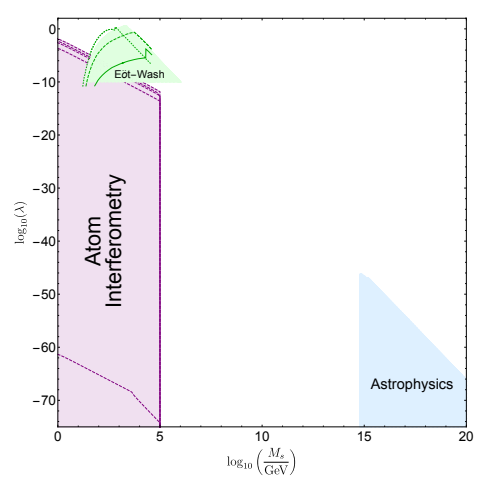

Fig. 2. Experimental constraints on the symmetron parameters $M$ and $\lambda$. The Eöt-Wash region corresponds to $\mu=2.4 \mathrm{meV}$; the outlines for values $\mu=\left\{10^{-4}, 10^{-3}, 10^{-2}\right\} \mathrm{eV}$ are shown by the solid, dashed, and dotted green lines respectively. The atom interferometry lines correspond to the regions excluded for $\mu=\left\{10^{-4}, 10^{-4.5}, 10^{-5}, 10^{-5}, 2.4 \times 10^{-3}\right\} \mathrm{eV}$ from top to bottom respectively, the latter value corresponding to the dark energy scale. The astrophysical bounds are insensitive to the value of $\mu$ for the values considered here.

cylinder filled with a low density gas surrounded by a dense metallic bore. In this case one can use a "bootstrapping" algorithm whereby the value of the field at the centre of the cylinder is left unknown, then solve the equations for the scalar field and finally impose that the value at the centre is indeed the one postulated initially. This yields a self-consistency condition which turns out to be the "resonance" criterion.

Outside the vacuum, and far inside the metal, the field settles at $\phi_{\infty}$ where the mass is $m_{\infty}$ and the field minimises the effective potential. Inside the vacuum we assume that the field takes a value $\phi_{0}$ at the centre of the cylinder for $r=0$. The mass there is defined as the second derivative of the effective potential $m_{0}$. Hence for $r \geqslant R$ we have

whilst inside

$$
\frac{d^{2} \phi}{d r^{2}}+\frac{1}{r} \frac{d \phi}{d r}-m_{\infty}^{2}\left(\phi-\phi_{\infty}\right)=0
$$

$$
\frac{d^{2} \phi}{d r^{2}}+\frac{1}{r} \frac{d \phi}{d r}-m_{0}^{2}\left(\phi-\phi_{0}\right)=V_{e f f}^{\prime}\left(\phi_{0}\right)
$$

where $V_{e f f}^{\prime}\left(\phi_{0}\right)=\frac{d V_{e f f}}{d \phi}\left(\phi_{0}\right) \neq 0$ if $\phi_{0}$ is not the minimum of the effective potential in vacuum. The solutions can be expressed in terms of Bessel and Neumann functions of zeroth order

$$
\begin{aligned}
& r<R \quad \phi=C J_{0}\left(i m_{0} r\right)+\phi_{0}-\frac{V_{e f f}^{\prime}\left(\phi_{0}\right)}{m_{0}^{2}} \\
& r \geqslant R \quad \phi=A\left(J_{0}\left(i m_{\infty} r\right)-i N_{0}\left(i m_{\infty} r\right)\right)+\phi_{\infty}
\end{aligned}
$$

where $A$ and $C$ are constants obtained by matching the field and its first derivative at $r=R$. When the mass inside the metallic bore is very large, i.e. $m_{\infty} R \gg 1$, this 
simplifies to

$$
\phi=\frac{\phi_{\infty}-\phi_{0}+\frac{V_{e f f}^{\prime}\left(\phi_{0}\right)}{m_{0}^{2}}}{J_{0}\left(i m_{0} R\right)} J_{0}\left(i m_{0} r\right)+\phi_{0}-\frac{V_{e f f}^{\prime}\left(\phi_{0}\right)}{m_{0}^{2}}
$$

for $r \leqslant R$. Evaluating this solution at $r=0$ gives us the resonance condition

$$
\phi_{\infty}-\phi_{0}=\frac{V_{e f f}^{\prime}\left(\phi_{0}\right)}{m_{0}^{2}}\left(J_{0}\left(i m_{0} R\right)-1\right) .
$$

Let us consider now two archetypical models. First for inverse power law chameleons and putting the density inside the cylinder to zero, we have $\phi_{\infty} \ll \phi_{0}$

$$
J_{0}\left(i m_{0} R\right)=n+2 .
$$

This is a resonance condition for $m_{0} R$ which should be of order one, i.e. the mass of the scalar field in the cylinder adapts itself to the radius of the cylinder. A simplified solution to this equation is $m_{0} R \sim 2 \sqrt{n+1}$ which is useful as an order of magnitude estimate. Using

$$
m_{0}^{2}=\frac{n(n+1) \Lambda^{n+4}}{\phi_{0}^{n+2}}
$$

one can easily evaluate the field inside the cavity.

For symmetrons, the solution to the resonance condition is more complex and can be deduced using the resonance condition written as

$$
J_{0}\left(i m_{0} R\right)=\frac{1+\frac{2 m_{0}^{2}}{m_{\text {vac }}^{2}}}{1-\frac{m_{0}^{2}}{m_{\text {vac }}^{2}}}
$$

where we have defined $m_{\mathrm{vac}}^{2}=2 \mu^{2}$ to be the mass of the symmetron in the symmetry breaking phase when to matter density is present, i.e. for $\phi_{\mathrm{vac}}=\frac{\mu}{\sqrt{\lambda}}$. This condition admits solutions only when

$$
m_{\mathrm{vac}} R \gtrsim 1,
$$

i.e. for values of $m_{\mathrm{vac}} \lesssim 1$ the only solution is $\phi=\phi_{\infty}=0$ inside the whole apparatus. When a solution exists we have

$$
m_{0} \sim m_{\mathrm{vac}}\left(1=\frac{1}{2} \sqrt{\frac{\pi m_{\mathrm{vac}} R}{2}} e^{-m_{\mathrm{vac}} R}\right)
$$

which implies that

$$
\phi_{0} \sim \phi_{\text {vac }}
$$

i.e. in the symmetron case and when the solution inside the chamber exists it is exponetially close to the vacuum solution in the absence of cavity. This is a very useful criterion which is used when analysing atomic interferometry data. 


\section{The Dilaton as a Worked out Example}

\subsection{The model}

We will now describe the laboratory constraints on different models from chameleons and symmetrons. In this section, we will work out another simple example: the environmentally dependent dilaton Brax et al.(2010b)Brax, van de Bruck, Davis, and Shaw This model has the advantage of being easily tractable and relatively well motivated. This will allow us to make completely explicit all the constraints which we have mentioned so far in a very simple setting. The potential for the dilaton is given by

$$
V(\phi)=V_{0} e^{-\lambda \phi / m_{\mathrm{P} 1}}
$$

where $V_{0}$ is an energy scale related to the dark energy of the Universe and $\lambda$ a numerical constant. This potential corresponds to the string theory dilaton potential in the strong coupling limit Damour et al.(2002)Damour, Piazza, and Veneziano The coupling function is inspired by the least coupling principle Damour and Polyakov(1994) where one assumes that in high density regions, the coupling of the dilaton to matter is driven to zero. It reads

$$
A(\phi)=1+\frac{A_{2}}{2 m_{\mathrm{Pl}}^{2}} \phi^{2} .
$$

where $A_{2} \gg 1$ to satisfy the solar system tests, see below. Notice the similarity with the symmetron with

$$
A_{2}=\frac{m_{\mathrm{Pl}}^{2}}{M^{2}} .
$$

In a dense environment with matter density $\rho$, the effective potential

$$
V_{\text {eff }}(\phi)=V_{0} e^{-\lambda \phi / m_{\mathrm{P} 1}}+\frac{A_{2} \rho}{2 m_{\mathrm{Pl}}^{2}} \phi^{2}
$$

admits a minimum at

$$
\phi_{\rho}=\frac{\lambda V_{0} m_{\mathrm{Pl}}}{A_{2} \rho} .
$$

where we have assumed that $\lambda \phi \ll m_{\mathrm{Pl}}$ as can be easily checked. The coupling to matter

$$
\beta(\phi)=m_{\mathrm{Pl}} \frac{\partial \ln A(\phi)}{\partial \phi}=A_{2} \frac{\phi}{m_{\mathrm{Pl}}}
$$

becomes

$$
\beta_{\rho}=\frac{\lambda V_{0} m_{\mathrm{Pl}}}{A_{2} \rho} .
$$

The mass at the minimum of the potential is given by

$$
m_{\rho}^{2}=\frac{\lambda^{2} V_{0}}{m_{\mathrm{Pl}}^{2}}+\frac{A_{2}}{m_{\mathrm{Pl}}^{2}} \rho .
$$


The value of the potential at the minimum is given by

$$
V_{\rho}=V_{0}+\frac{\lambda^{2} V_{0}^{2}}{2 A_{2} \rho}
$$

which is always close to $V_{0}$ and we choose to tune it to the value of the vacuum energy now

$$
V_{0}=3 \Omega_{\Lambda 0} m_{\mathrm{Pl}}^{2} H_{0}^{2}
$$

where $\Omega_{\Lambda 0} \sim 0.7$. Notice that the coupling to matter in the cosmological vacuum is given by

$$
\beta_{0}=\lambda \frac{\Omega_{\Lambda 0}}{\Omega_{m 0}} .
$$

which can be arbitrarily small with $\lambda$.

The strongest constraint on the dilaton models comes from the Laser Lunar Ranging experiment Williams et al.(2012)Williams, Turyshev, and Boggs giving

$$
\eta \equiv 2 \frac{\left|a_{\oplus}-a_{\text {moon }}\right|}{a_{\oplus}+a_{\text {moon }}} \lesssim 10^{-13}
$$

for the moon and the earth in the background of the sun. This is related to the way screened bodies like the sun, the earth and the moon (because of their large densities) couple to the scalar field

$$
\eta \sim Q_{\odot}\left|Q_{\oplus}-Q_{\text {moon }}\right|
$$

where the charge $Q_{A}=\beta$ is the coupling to matter in the environment of an object for unscreened bodies and

$$
Q_{A}=\frac{\phi_{G}}{2 m_{\mathrm{Pl}} \Phi_{A}}
$$

for a screened body of Newtonian potential $\Phi_{A}$ at its surface and embedded in the environment where the scalar field takes a value $\phi_{G}$. For the LLR experiment, $\phi_{G}$ is the field value in the galactic medium of density $\rho_{G} \sim 10^{6} \rho_{m 0}$ where $\rho_{m 0}$ is the cosmological Cold Dark Matter density. This implies that

$$
\eta \sim 10^{-1} \frac{\phi_{G}^{2}}{m_{\mathrm{Pl}} \Phi_{\oplus}^{2}}
$$

where $\Phi_{\oplus} \sim 10^{-9}, \Phi_{\odot} \sim 10^{-6}, \Phi_{\text {moon }} \sim 10^{-11}$. This leads to the bound

$$
\frac{A_{2}}{\lambda} \gtrsim \Phi_{\oplus}^{-1} \sim 10^{9}
$$

and finally for the mass of the dilaton in the cosmological background

$$
m_{0} \gtrsim \sqrt{\lambda} \Phi_{\oplus}^{-1 / 2} H_{0}
$$

which for $\lambda=1$ becomes $\operatorname{Brax}$ and Davis(2015)

$$
m_{0} \gtrsim 34500 H_{0} .
$$

This implies that the effects of the dilaton on the growth of cosmic structure would occur on scales less than $1 \mathrm{Mpc}$. 
16 Authors' Names

\subsection{Laboratory experiments}

The first step in modeling the behaviour of the dilaton in an experimental context is to solve for the field profile between two infinitely thick plates located at $|z| \geqslant d$ where $2 d$ is the inter-plate distance. Inside the plate which has a density $\rho_{c}$ the field converges to $\phi_{c}$ deep inside. Between the plates, the field profile would be given by $\phi_{b}$ for the density $\rho_{b}$ if the distance $d$ were infinite. As $d$ is finite, the field reaches a smaller value $\phi_{0}$ for $z=0$. As $\rho_{c} \gg \rho_{b}$ we can approximate $\phi_{c} \sim 0$ and the field profile between the plates is given by

$$
\phi^{\prime 2}(z)=2\left(V_{\text {eff }}(\phi)-V_{\text {eff }}\left(\phi_{0}\right)\right)
$$

where a very good approximation is given by

$$
V_{\text {eff }}(\phi) \sim V_{b}+\frac{1}{2} m_{b}^{2}\left(\phi-\phi_{b}\right)^{2}
$$

depending on the density $\rho_{b}$. Explicitly this gives

$$
\phi(z)=\phi_{b}\left(1-\frac{\cosh m_{b} z}{\cosh m_{b} d}\right) .
$$

In particular we have

$$
\phi_{0}=\phi_{b}\left(1-\frac{1}{\cosh m_{b} d}\right)
$$

which converges to $\phi_{b}$ when $m_{b} d \gg 1$.

It turns out that the pressure exerted by one of the plates on the other one, i.e. the scalar equivalent to the Casimir effect, is given by Brax and Davis(2015)

$$
\frac{\Delta F}{A}=V_{\mathrm{eff}}\left(\phi_{b}\right)-V_{\mathrm{eff}}\left(\phi_{0}\right)
$$

depending on the potential difference between the energy stored in the field configuration in the absence and in the presence of the plates. In the dilaton case, this becomes

$$
\frac{\Delta F}{A}=-\frac{m_{b}^{2} \phi_{b}^{2}}{2 \cosh ^{2} m_{b} d}
$$

which is attractive. There are two clear regimes. When $m_{b} d \gg 1$, we have

$$
\frac{\Delta F}{A}=-\frac{m_{b}^{2} \phi_{b}^{2}}{2} e^{-2 m_{b} d}
$$

corresponding to a Yukawa suppressed interaction (as the distance is $2 d$ ) whilst for $m_{b} d \ll 1$ we have

$$
\frac{\Delta F}{A}=-\frac{m_{b}^{2} \phi_{b}^{2}}{2}
$$

corresponding to a pressure given by the amount of energy stored by the scalar field in vacuum.

The most stringent laboratory constraint on dilatons springs from the negative experimental results on the 
existence of short range scalar interactions by the Eöt-Wash experiment in Seattle Kapner et al.(2007)Kapner, Cook, Adelberger, Gundlach, Heckel, Hoyle, and Swanson The measured torque between two torsion pendulum is given by Brax et al.(2008)Brax, van de Bruck, Davis, and Shaw

$$
T=a_{\theta} \int_{d}^{\infty} d x\left|\frac{\Delta F}{A}(x)\right|
$$

where $a_{\theta}$ is a constant depending on the experiment and the Eötwash constraint is $T \leqslant a_{\theta} \Lambda_{T}^{3}$ where $\lambda_{T}=0.35 \Lambda$, where $V_{0}=\Lambda^{4}$, for $d=55 \mu \mathrm{m}$. As long as $d m_{b} \ll 1$, the torque is given by

$$
T \sim a_{\theta} \frac{\lambda^{2} V_{0}^{2}}{2 A_{2} \rho_{b} m_{b}} \sim a_{\theta} \frac{\lambda^{2} V_{0}}{2 m_{\mathrm{Pl}}^{2} m_{b}^{3}}
$$

coinciding with (5.6) in Brax and Davis(2015) As a result we find that

$$
m_{b} \gtrsim H_{0}^{2} \Lambda
$$

and using the background density $\rho_{b} \sim 10^{-27} \mathrm{GeV}^{4}$, this gives the weak constraint on the mass $m_{0}$ in the cosmological background Brax and Davis(2015)

$$
m_{0} \gtrsim 55 H_{0}
$$

The same type of techniques can be applied to the chameleon and symmetron models which we will not detail and refer only to the existing literature. The main difference in the chameleon and symmetron case is that one must take into account the electrostatic shield between the two plates implying that the torque is reduced by the Yukawa suppression

$$
T \rightarrow e^{-m_{s} D} T
$$

where $m_{s}$ is the scalar mass in the shield and $D$ its width. For chameleons, this is responsible for the loss of sensitivity of the Eötwash experiment at very large coupling $\beta$ where the mass becomes large and the torque is essentially zero.

\section{Tomographic parameterisation}

The models that we have considered, i.e. chameleons, symmetrons and dilatons can all be described using an implicit definition of the coupling function $A(\phi)$ and $V(\phi)$. This method, which is called tomographic, uses the explicit link between the density dependence of the minimum of the effective potential as a function of the matter density in the environment and the shape of the potential and coupling functions. This applies to inverse power law chameleon models, symmetrons and dilatons which are all scalar-tensor theories described by the Lagrangian

$$
S=\int d^{4} x \sqrt{-g}\left(\frac{R}{16 \pi G_{N}}-\frac{(\partial \phi)^{2}}{2}-V(\phi)\right)+S_{m}\left(\psi, A^{2}(\phi) g_{\mu \nu}\right)
$$


where $A(\phi)$ is a function which defines the coupling between matter fields $\psi$ and the scalar $\phi$. The coupling to matter itself is

$$
\beta(\phi)=m_{\mathrm{Pl}} \frac{d \ln A(\phi)}{d \phi} .
$$

The scalar field dynamics are determined by an effective potential which takes into account the presence of the conserved matter density $\rho$ in the environmenta

$$
V_{\text {eff }}(\phi)=V(\phi)+(A(\phi)-1) \rho .
$$

where the -1 is introduced for convenience. Chameleon-like theories, e.g. symmetrons and dilatons, are such that the effective potential admits a minimum as a function of the density where the mass function $m(\rho)$ and the coupling $\beta(\rho)$ at the minimum of the effcetive potential become density dependent Brax et al.(2012a)Brax, Davis, Li, and Winther Brax et al.(2012b)Brax, Davis, and Li It is easier in view of comparing with cosmological tests to characterise the functions $m(\rho)$ and $\beta(\rho)$ using the time evolution of the matter density of the Universe $\rho(a)=\frac{\rho_{0}}{a^{3}}$ where $a \leqslant 1$ is the scale factor of the Universe whose value now is $a_{0}=1$. This allows one to parameterise all chameleon-like theories using simply the $a$ dependence of $\beta(a)$ and $m(a)$. Parametrically we have

$$
\frac{\phi(a)-\phi_{c}}{m_{\mathrm{Pl}}}=9 \Omega_{m 0} H_{0}^{2} \int_{a_{c}}^{a} d a \frac{\beta(a)}{a^{4} m^{2}(a)},
$$

where the Hubble rate now is $H_{0} \sim 10^{-43} \mathrm{GeV}$ and the matter fraction is $\Omega_{m 0} \sim$ 0.27 . and the mass function is defined as the mass at the minimum of the effective potential $\phi(\rho(a))$

$$
m^{2}(a)=\left.\frac{d^{2} V_{\mathrm{eff}}}{d \phi^{2}}\right|_{\phi=\phi(\rho(a))} .
$$

Similarly the coupling is

$$
\beta(a)=\left.m_{\mathrm{Pl}} \frac{d \ln A}{d \phi}\right|_{\phi=\phi(\rho(a))} .
$$

and the potential value is given by

$$
V(a)-V_{c}=-27 \Omega_{m 0}^{2} H_{0}^{4} \int_{a_{c}}^{a} d a \frac{\beta^{2}(a) m_{\mathrm{Pl}}^{2}}{a^{7} m^{2}(a)} .
$$

This implicit parameterisation of $V(\phi)$ and $A(\phi)$ is obtained directly from from $m(a)$ and $\beta(a)$, i.e. one can reconstruct the potential by eliminating $a$ between (67) and 70 .

Familiar models can be easily described using this method. Chameleons with a potential of the type

$$
V(\phi)=\Lambda^{4}+\frac{\Lambda^{4+n}}{\phi^{n}}+\ldots
$$

\footnotetext{
a The conserved energy density is related to the density defined a $\rho_{E}=-T_{0}^{0}$ in the Einstein frame
} as $\rho_{E}=A \rho$ 
where $n>0$, and $\Lambda \sim 10^{-3} \mathrm{eV}$ is the cosmological vacuum energy now, and the coupling function is $A(\phi)=\exp \left(\frac{\beta \phi}{m_{\mathrm{Pl}}}\right)$, can be reconstructed using $\beta(a)=\beta$ and

$$
m(a)=m_{0} a^{-r}
$$

where $r=\frac{3(n+2)}{2(n+1)}$. The mass scale $m_{0}$ is determined by

$$
m_{0}^{2(n+1)}=\frac{(n+1)^{n+1}}{3 n} \frac{\left(3 \beta \Omega_{m 0} H_{0}^{2} m_{\mathrm{Pl}}\right)^{n+2}}{\Lambda^{4+n}} .
$$

For $f(R)$ models in the large curvature regime

$$
f(R)=\Lambda_{0}+R-\frac{f_{R_{0}}}{n} \frac{R_{0}^{n+1}}{R^{n}} .
$$

where $\Lambda_{0}$ is the cosmological constant and $R_{0}$ is the present day curvature, we have $\beta(a)=1 / \sqrt{6}$ and the mass function

$$
m(a)=m_{0}\left(\frac{4 \Omega_{\Lambda 0}+\Omega_{m 0} a^{-3}}{4 \Omega_{\Lambda 0}+\Omega_{m 0}}\right)^{(n+2) / 2}
$$

where the mass on large cosmological scale is given by $m_{0}=H_{0} \sqrt{\frac{4 \Omega_{\Lambda 0}+\Omega_{m 0}}{(n+1) f_{R_{0}}}}$, and $\Omega_{\Lambda 0} \approx 0.73$ is the dark energy fraction now Brax et al.(2012a)Brax, Davis, Li, and Winther When $a \ll 1$, i.e. physical situations where the environment is dense, the mass dependence on $a$ is a power law $m(a) \sim m_{0} a^{-r}$ where $r=\frac{3(n+2)}{2}$. Dilatons are described by

$$
\beta(a)=\beta_{0} a^{3}
$$

and the mass function

$$
m^{2}(a)=3 A_{2} \frac{H_{0}^{2}}{a^{3}} .
$$

Finally the symmetrons are defined by the potential

$$
V(\phi)=V_{0}+\frac{\lambda}{4} \phi^{4}-\frac{\mu^{2}}{2} \phi^{2}
$$

and a coupling function

$$
A(\phi)=1+\frac{\beta_{\star}}{2 \phi_{\star} m_{\mathrm{Pl}}} \phi^{2}
$$

where the transition from the minimum of the effective potential at the origin to a non-zero value happens for a density $\rho_{\star}$. Defining

$$
m_{\star}=\sqrt{2} \mu, \phi_{\star}=\frac{2 \beta_{\star} \rho_{\star}}{m_{\star}^{2} m_{\mathrm{Pl}}}, \lambda=\frac{\mu^{2}}{\phi_{\star}^{2}}
$$

where $\rho_{\star}=\frac{\rho_{m 0}}{a_{\star}^{3}}$, the model can be reconstructed using

$$
m(a)=m_{\star} \sqrt{1-\left(\frac{a_{\star}}{a}\right)^{3}}
$$


and

$$
\beta(a)=\beta_{\star} \sqrt{1-\left(\frac{a_{\star}}{a}\right)^{3}}
$$

for $a>a_{\star}$ and $\beta(a)=0$ for $a<a_{\star}$. For all these models, the comparison with laboratory tests can be done using the same techniques as the ones outlined in the previous section for the dilaton.

\section{Vainshtein mechanism}

The Vainshtein screening is difficult to constrain with laboratory tests, at least for cosmologically interesting models. Theories which contain Vainshtein screening rely on non-linear kinetic terms. This means that the variation in the field is extremely slow, and so the field can only respond to spatial variations in the local density over long distance scales. As with other types of screening the fifth force can be suppressed by the environment so that, for example, fifth forces in the solar system are screened by the galactic density. However, unlike theories with chameleon screening, fifth forces with Vainshtein screening do not respond quickly enough to changes in density that they can be unscreened in a laboratory vacuum.

$$
\text { It is still possible to place }
$$
bounds on theories with Vainshtein screening from laboratory tests, for example on the Galileon models Brax et al.(2011a)Brax, Burrage, and Davis However these bounds are weak, and the relationship between the parameters that can be constrained in these experiments, and the fundamental parameters relevant for the cosmology is non-trivial.

Both Eöt-Wash and Casimir experiments use planar geometry. Considering the Galileon with planar symmetry one find the non-linear terms cancel order by order, so the fifth force is completely unscreened Bloomfield et al.(2015)Bloomfield, Burrage, and Davis One finds the ratio of the Galileon to Newtonian force is

$$
\frac{F_{\phi}}{F_{G}}=2 \beta^{2} .
$$

Similarly one can compute the screening for a cylindrical object and show the screening is reduced. Here the ratio of the Galileon to Newtonian force for the cubic Galileon is

$$
\frac{F_{\phi}}{F_{G}}=2 \beta^{2} \frac{r}{r_{v}},
$$

compared to the spherically symmetric case of

$$
\frac{F_{\phi}}{F_{G}}=2 \beta^{2}\left(\frac{r}{r_{v}}\right)^{3 / 2} .
$$

In both cases $r_{v}$ is the Vainshtein radius.Thus searching for Galileons in planar symmetric objects would yield a strong fifth force. The Galileon force around a 
plate of density $\rho$ and thickness $\Delta$ when there is no screening is

$$
\phi^{\prime}=\frac{\rho}{2 c_{2}}\left\{\begin{array}{cc}
\Delta & r>\Delta / 2 \\
2 z & \Delta / 2>r>-\Delta / 2 \\
-\Delta & -\Delta / 2>r
\end{array},\right.
$$

where we have approximated the plate as a one-dimensional object and imposed continuity at the boundary. When one considers laboratory experiments one needs to consider the environment as well as the physical set-up. Both Eöt-Wash and Casimir experiments are performed on Earth, which must be taken into consideration since the radius of the Earth is less than the typical Vainshtein radius. In order to take this into account one writes $\phi(\vec{x})=\phi_{\oplus}(r)+\delta \phi(z)$, where $\delta \phi$ is the perturbation due to the plates used in the experiment and the background field due to the Earth is $\phi_{\oplus}(r)$. Substituting this into the equation of motion one find that it depends on a function $Z(r)$ which is approximately constant at $r=R_{\oplus}$,

$$
Z_{\oplus}=\frac{R_{\oplus}}{\pi_{\oplus}^{\prime}}\left[\frac{2 \rho_{\oplus}}{3}+8 c_{4}\left(\frac{\pi_{\oplus}^{\prime}}{R_{\oplus}^{3}}\right)^{3}+32 c_{5}\left(\frac{\pi_{\oplus}^{\prime}}{R_{\oplus}^{3}}\right)^{4}\right] .
$$

The exact size of $Z_{\oplus}$ depends on the parameters $c_{4}$ and $c_{5}$. Laboratory experiments can be used to constrain the combination of coefficients in $Z_{\oplus}$. Of-course the effect of the cavity needs to be taken into account as well. However, after detailed calculation it was found that in the background field of the Earth the cavity and plates behave as a linear theory with $c_{2}$ replaced by $Z_{\oplus}$.

The experiments we consider consist of two plates, aligned perpendicular to the $z$-direction, whose extent in the $x, y$-directions is much larger that their separation so we can approximate them as infinite. The plates have density $\rho$. The lower edge of one plate and the upper edge of the other is positioned at $z=d$ and $z=-d$ respectively, and the plates have width $\Delta$. Therefore $\phi^{\prime}$, the strength of the Galileon force due to the configuration, is given by

$$
\phi^{\prime}=\frac{\rho}{Z_{\oplus}}\left\{\begin{array}{lc}
z-d & d<z<d+\Delta \\
0 & -d<z<d \\
z+d & -(d+\Delta)<z<-d
\end{array},\right.
$$

where we have imposed continuity of $\pi^{\prime}$ at the boundary of the plates, and $\pi^{\prime}(z)=$ $-\pi^{\prime}(-z)$. The approximation that the plates are infinite is valid whenever their extent in the $x, y$-directions is much larger than the distance $2 d$ between the plates. Applying this to the Eöt-Wash experiment described earlier we find the torque induced by the Galileon is

$$
T=\frac{\rho^{2} \Delta^{3}}{6 Z_{\oplus}} a_{T},
$$

where $a_{T}=d A / d \theta$ is a constant which depends on the experimental setup $a_{T}=$ $3 \times 10^{-3} \mathrm{~m}^{2}$. The width of the plates is $\Delta=1 \mathrm{~mm}$ and the plates are made of 
molybdenum with a density $\rho=10.28 \mathrm{gcm}^{-3}$. The constraint on the Galileon force thus becomes

$$
\begin{aligned}
Z_{\oplus} & >6.05 \times 10^{40} \mathrm{GeV}^{2}, \\
& >\left(20 m_{P}\right)^{2},
\end{aligned}
$$

which translates into a constraint on the coupling

$$
\beta<0.05 \text {. }
$$

Hence we find that in the context of the Eöt-Wash experiment, the Galileon force between the plates must be much weaker than the gravitational one. This sets a bound on a previously unconstrained combination of the Galileon parameters.

For Casimir experiments the formalism described above is not relevant to the experiments

of Decca et al Decca et al.(2007)Decca, Lopez, Fischbach, Klimchitskaya, Krause, and Mostepanenko Chen et al.(2016)Chen, Tham, Krause, Lopez, Fischbach, and Decca where the force between a plate and a sphere is measured, It can be applied to the force between two parallel plates Bressi et al.(2002)Bressi, Carugno, Onofrio, and Ruosd|Bressi et al.(2000)Bressi, Carugno, Galvani, Onofrio, and Ruoso However, following the procedure outlined for Eöt-Wash we find the Galileon force could not be detected in these experiments. In Brax et al.(2010a)Brax, van de Bruck, Davis, Shaw, and Iannuzzi|Almasi et al.(2015)Almasi, Brax, Iannuzzi, and Sedmik it was proposed that a modified parallel plate Casimir experiment could be used to search for the Chameleon by exploiting the change in the Chameleon force as the density of the inter-plate medium changes. As the Galileon force also depends on the local energy density this experiment could also provide useful constraints on the Galileon model.

\section{Coupling to Photons}

Conformally coupled scalar fields do not, classically, interact with photons. This can be seen directly from the conformal invariance of the photon terms of the standard model Lagrangian;

$$
\tilde{g}_{\mu \nu}=A^{2}(\phi) g_{\mu \nu}
$$

where $g_{\mu \nu}$ is the Einstein frame metric. Assuming no coupling between the scalar and photons in the Jordan frame,

$$
S_{F}=-\frac{1}{4} \int d^{4} x \sqrt{-\tilde{g}} \tilde{g}^{\mu \rho} \tilde{g}^{\nu \lambda} F_{\mu \nu} F_{\rho \lambda}
$$

with $F_{\mu \nu}=\partial_{\mu} A_{\nu}-\partial_{\nu} A_{\mu}$. We see that in the Einstein frame

$$
S_{F}=-\frac{1}{4} \int d^{4} x \sqrt{-g} g^{\mu \rho} g^{\nu \lambda} F_{\mu \nu} F_{\rho \lambda}
$$

which makes explicit the scale invariance of the photon Lagrangian.

However there is not reason to forbid a interactions between the scalar and photons, and there are a number of reasons to expect such a coupling to emerge, which we will describe in the next section. 


\subsection{Quantum Coupling}

Whilst a classical Weyl rescaling does not induce a coupling between the scalar and photons, it can be shown that, after quantisation of the fields such a coupling does indeed emerge Brax et al.(2011b)Brax, Burrage, Davis, Seery, and Weltman The conformal invariance of the photon Lagrangian is broken by a quantum anomaly which comes from the rescaling of the fermions $\psi \rightarrow A^{3 / 2}(\phi) \psi$ from the Jordan to the Einstein frame and the subsequent change of the measure in the path integral defining the quantum theory.

Additionally, if there are heavy charged fermions beyond the Standard Model then the fermions, which will couple directly to the scalar, will be able to mediate interactions between the scalar and photons through a triangle loop diagram. Integrating these heavy fermions out, leaves a low energy effective theory which possesses a contact interaction between the conformally coupled scalar and two photons Brax et al.(2011b)Brax, Burrage, Davis, Seery, and Weltman Nitti and Piazza(2012) the Einstein frame action picks up a field dependent coupling to photons

$$
S_{F}=-\frac{1}{4} \int d^{4} x \sqrt{-g} B(\phi) g^{\mu \rho} g^{\nu \lambda} F_{\mu \nu} F_{\rho \lambda}
$$

such that the dimensionless constant controlling the strength of the coupling

$$
\beta_{\gamma}=m_{\mathrm{Pl}} \frac{\partial \ln B}{\partial \phi}
$$

becomes

$$
\beta_{\gamma}=\left(3 N_{f}+\frac{N_{f}^{>}}{3}\right) \frac{\alpha}{4 \pi} \beta_{m}
$$

where we have introduced

$$
\beta_{m}=m_{\mathrm{Pl}} \frac{\partial \ln A}{\partial \phi} .
$$

Here $N_{f}$ is the total number of fermions in the model, for instance the fermions of the standard model, and $N_{f}^{>}$the number of fermions which have been integrated out, e.g. the fermions with a mass at the grand unification scale. Of course as $N_{f}^{>}$ is not known, the precise value of $\beta_{m}$ cannot be inferred and in general is taken to be a non-vanishing parameter of the model.

\subsection{Photon-scalar mixing}

The presence of the coupling $\beta_{\gamma}$ implies that the photons and scalars mix and therefore that the mass eigenstates do not coincide with the propagating fields. Physically this can be seen as the effect such that a propagating photon has a nonzero probability of becoming a scalar before reverting back to a photon state. Hence this will have two consequences: the effective speed of the photons is affected by the coupling $\beta_{\gamma}$ as the photon wave function picks up a non-vanishing phase shift after a finite distance and the amplitude of the wave function is also altered. This 
happens when a magnetic field $\vec{B}$ is present where the scalar-Maxwell equations become

$$
\square \vec{A}=\frac{\beta_{\gamma}}{m_{\mathrm{Pl}}} \vec{\nabla} \phi \wedge \vec{B}
$$

and

$$
\square \phi-m^{2} \phi=\frac{\beta_{\gamma}}{m_{\mathrm{Pl}}} \vec{B} \cdot \vec{\nabla} \wedge \vec{A}
$$

Assuming that the magnetic field is perpendicular to the propagation of the photon, only the parallel polarisation of the photon along the magnetic field mixes with the scalar. Define the mixing angle as

$$
\tan 2 \theta=\frac{2 B \omega}{M m^{2}}
$$

where $\beta_{\gamma}=\frac{m_{\mathrm{P} 1}}{M}$ and $m$ is the mass of the scalar in the magnetised region where the photon propagates. The energy of the initial photon is $\omega$ and the two propagating modes of the system have momenta along the photon direction Brax et al.(2007b)Brax, van de Bruck, Davis, Mota, and Shaw

$$
k_{-}=\omega+\frac{m^{2} \theta^{2}}{2 \omega}, \quad k_{+}=\omega-\frac{m^{2}}{2 \omega}
$$

where we assume that the mixing angle $\theta \ll 1$ is small. After a distance $z$, the photon wave function becomes

$$
a_{\|}(z)=(1-a(z)) \cos (\omega z+\delta)
$$

where

$$
a(z)=2 \theta^{2} \sin ^{2} \frac{m^{2} z}{4 \omega}
$$

and

$$
\delta=\theta^{2} \frac{m^{2}}{2 \omega} z-\theta^{2} \sin \frac{m^{2} z}{2 \omega} .
$$

This is the result for the free propagation of photons. Inside a cavity, the photons are reflected $N$ times before leaving and being detected. Introducing the coherence length

$$
z_{c o h}=\frac{2 \omega}{m^{2}}
$$

and the number of coherent passes $P$ in a cavity of length $L$

$$
P L=2 \pi z_{c o h}
$$

the overall change of amplitude and phase shift become Brax et al.(2007b)Brax, van de Bruck, Davis, Mota, and Shaw

$$
a_{T}=\theta^{2}, \delta_{T}=\frac{\pi N \theta^{2}}{P} .
$$


It is possible to measure the rotation of the initial polarisation per pass and the induced ellipticity of the polarisation which does not remail linear

$$
\text { rotation } / \text { pass }=\frac{\theta^{2}}{2 N}, \text { ellipticity } / \text { pass }=\frac{\pi \theta^{2}}{2 P} .
$$

Laser experiments constrain these observables and therefore give constraints on the coupling to photons.

\subsection{Scalar reflection}

As the mass of the scalar jumps from a low value inside the cavity to a large value inside the cavity's walls, the scalar wave function is distorted by the presence of the wall. This induces another phase shift compared to the one calculated in the previous section. The phenomenology of scalars inside cavities has been thoroughly investigated for chameleons and we refer to Brax et al.(2007b)Brax, van de Bruck, Davis, Mota, and Shaw for details. Here we will elaborate on the dilaton case as the calculations are simpler.

For a dilaton in a cavity with a vacuum density $\rho_{b}$ and walls of density $\rho_{c}$ and associated minima of the effective potential $\phi_{b, c}$, the static profile of the scalar is obtained by solving

$$
\phi^{\prime 2}(z)=V_{\text {eff }}(\phi)-V_{\text {eff }}\left(\phi_{b}\right)
$$

for $z \geqslant 0$ where the wall is at $z=0$ here. For a dilaton we find

$$
\phi(z)=\phi_{0}+\left(\phi_{b}-\phi_{0}\right)\left(1-e^{-m_{b} z}\right)
$$

where $\phi_{0}=\phi(0) \approx \phi_{c}$ as $\rho_{c} \gg \rho_{b}$. The mass of the scalar evolves away from the wall as

$$
m^{2}(z)=m_{b}^{2}\left(1-\frac{\lambda^{3} V_{0}}{A_{2} \rho_{b}} \frac{\phi(z)-\phi_{b}}{m_{\mathrm{Pl}}}\right)=m_{b}^{2}\left(1+\frac{\lambda^{3} V_{0}}{A_{2} \rho_{b}} \frac{\phi_{b}-\phi_{c}}{m_{\mathrm{Pl}}} e^{-m_{b} z}\right)
$$

i.e. the mass decreases exponentially. Let us consider a scalar wave which is a solution of

$$
\frac{d^{2} \delta \phi}{d z^{2}}+\left(\omega^{2}-m^{2}(z)\right) \delta \phi=0 .
$$

There are three regions to consider. Inside the cavity where $m(z) \sim m_{b}$, the scalar propagates as a wave alongside the photons. In the walls when $\omega \leqslant m_{c}$, the field is attenuated and the scalar is therefore reflected. This has been tested in afterglow experiments where a laser beam is turned off and one expects that the trapped scalars in the cavity will regenerate photons which would then be seen as afterglows. Finally when $\omega$ is not much larger than $m_{b}$, the scalar is reflected at $z_{\omega}$ where $m\left(z_{\omega}\right)=\omega$ implying that this induces a phase shift compared to the photons, i.e. the scalar is reflected before the photons. This plays a crucial role for chameleons and was taken into account in Brax et al.(2007b)Brax, van de Bruck, Davis, Mota, and Shaw 


\subsection{Experimental results}

There are three types of experiments which must be taken into account. We will give the bounds for the chameleon model as this is the most studied case. In the symmetron case, the phenomenology has not been worked out and should be closer to the dilaton case presented in the previous section than to the chameleon case. The first type of experiments measure the induced ellipticity and rotation angle of a laser beam where a transverse magnetic field is present inside a high quality cavity. From the 2007 PVLAS results Zavattini et al.(2008) with $N=45000$ passes, the total ellipticity is constrained to be

$$
\text { ellipticity } \leqslant 1.4 \times 10^{-8}
$$

and the rotation

$$
\text { rotation } \leqslant 10^{-8}
$$

with a cavity length of $L=1 \mathrm{~m}$, a beam with $\omega=1.17 \mathrm{eV}$ and a magnetic field

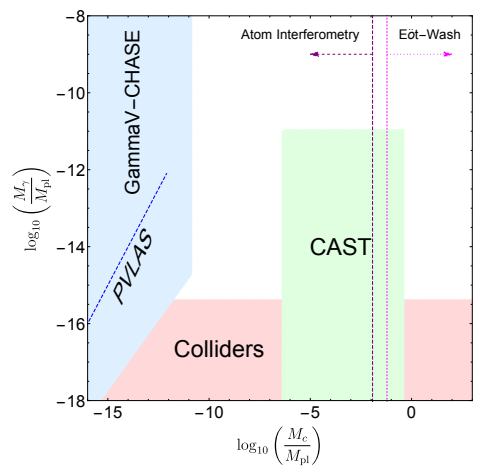

Fig. 3. Experimental constraints on the coupling of the chameleon to photons as a function of the chameleon coupling to matter, for the chameleon potential $V(\phi)=\Lambda^{5} / \phi$.

$B=2.3 \mathrm{~T}$. When $\beta_{\gamma}=\frac{M_{\mathrm{P} 1}}{M_{\gamma}}$ and $\beta=\frac{M_{\mathrm{P} 1}}{M_{c}}$ are such that $M_{\gamma}=M_{c}$ this yields $M_{\gamma} \geqslant 2 \times 10^{6} \mathrm{GeV}$. The ALPS experiment at DESYEhret et al.(2010) has performed a light-shining-through-walls experiment, where a laser beam with $\omega=2.33 \mathrm{eV}$ faces a wall after $L=4.3 \mathrm{~m}$ in a magnetic field $B=5 \mathrm{~T}$. The probability that a photon converts into a scalar after $L$

$$
P_{\gamma \rightarrow \phi}=\sin ^{2} 2 \theta \sin ^{2} \lambda \omega L
$$

where $\lambda=\frac{m^{2}}{2 \omega^{2}}\left(1+\tan ^{2} 2 \theta\right)$ is constrained to be

$$
P_{\gamma \rightarrow \phi} \leqslant 2.08 \times 10^{-25} .
$$


This competes with the CHASE experiment $\underline{\operatorname{Steffen}(2010)}$ where the afterglow phenomenon has been investigated and a bound $M_{\gamma} \geqslant 3 \times 10^{7} \mathrm{GeV}$ was found. Similarly inside the sun in the tachocline where the solar magnetic field is assumed to emerge, chameleons could be created and then observed with the CAST experiment where X-rays would be back-converted from solar chameleons which would have escaped the sun Brax and Zioutas(2010) Brax et al.(2012c)Brax, Lindner, and Zioutas The production and regeneration of X-rays depend on the coupling to photons $\beta_{\gamma}$. All these constraints are summarised in figure 1.

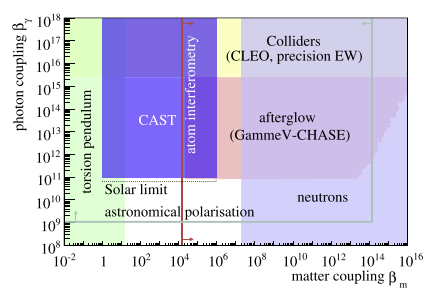

Fig. 4. Experimental constraints on chameleons with $n=1$ in the $\beta_{m}-\beta_{\gamma}$ plane Anastassopoulos et al.(2015)

\section{Conclusions}

In summary, laboratory experiments have a huge amount to tell us about possible modifications of gravity. For theories with screening, these experiments necessarily probe the non-linear parts of the theory, although we have shown how the effects of local screening due to the galactic environment can be 'un-screened' in a laboratory vacuum chamber. As they probe the non-linear regime, there is no model independent way to connect these constraints to the parametrized linear and quasi-linear theories used to obtain cosmological constraints. However, once a model is specified, we have shown the power of combining experimental searched on all scales from the sub-atomic to the cosmological.

\section{References}

Joyce et al.(2015)Joyce, Jain, Khoury, and Trodden. A. Joyce, B. Jain, J. Khoury, and M. Trodden, Phys. Rept. 568, 1 (2015), 1407.0059

Bull et al.(2016). P. Bull et al., Phys. Dark Univ. 12, 56 (2016), 1512.05356.

Burrage and Sakstein(2016). C. Burrage and J. Sakstein, JCAP 1611, 045 (2016), 1609. 01192

Burrage and Sakstein(2017). C. Burrage and J. Sakstein (2017), 1709.09071.

Elder et al.(2016)Elder, Khoury, Haslinger, Jaffe, Mller, and Hamilton.

B. Elder, J. Khoury, P. Haslinger, M. Jaffe, H. Mller, and P. Hamilton, Phys. Rev. D94, 044051 (2016), 1603.06587. 
Schlgel et al.(2016)Schlgel, Clesse, and Fzfa. S. Schlgel, S. Clesse, and A. Fzfa, Phys. Rev. D93, 104036 (2016), 1507.03081.

Burrage et al.(2015)Burrage, Copeland, and Hinds. C. Burrage, E. J. Copeland, and E. A. Hinds, JCAP 1503, 042 (2015), 1408.1409.

Burrage and Copeland(2016). C. Burrage and E. J. Copeland, Contemp. Phys. 57, 164 (2016), 1507.07493

Hamilton et al.(2015)Hamilton, Jaffe, Haslinger, Simmons, Mller, and Khoury.

P. Hamilton, M. Jaffe, P. Haslinger, Q. Simmons, H. Mller, and J. Khoury, Science 349, 849 (2015), 1502.03888.

Burrage et al.(2016)Burrage, Kuribayashi-Coleman, Stevenson, and Thrussell. C. Burrage, A. Kuribayashi-Coleman, J. Stevenson, and B. Thrussell, JCAP 1612, 041 (2016), 1609.09275.

Jaffe et al.(2017)Jaffe, Haslinger, Xu, Hamilton, Upadhye, Elder, Khoury, and Mller. M. Jaffe, P. Haslinger, V. Xu, P. Hamilton, A. Upadhye, B. Elder, J. Khoury, and H. Mller, Nature Phys. 13, 938 (2017), 1612.05171.

Adelberger et al.(2003)Adelberger, Heckel, and Nelson. E. G. Adelberger, B. R. Heckel, and A. E. Nelson, Ann. Rev. Nucl. Part. Sci. 53, 77 (2003), hep-ph/0307284

Kapner et al.(2007)Kapner, Cook, Adelberger, Gundlach, Heckel, Hoyle, and Swanson. D. J. Kapner, T. S. Cook, E. G. Adelberger, J. H. Gundlach, B. R. Heckel, C. D. Hoyle, and H. E. Swanson, Phys. Rev. Lett. 98, 021101 (2007), hep-ph/0611184.

Lambrecht et al.(2005)Lambrecht, Nesvizhevsky, Onofrio, and Reynaud. A. Lambrecht, V. V. Nesvizhevsky, R. Onofrio, and S. Reynaud, Class. Quant. Grav. 22, 5397 (2005).

Brax et al.(2008)Brax, van de Bruck, Davis, and Shaw. P. Brax, C. van de Bruck, A.-C. Davis, and D. J. Shaw, Phys. Rev. D78, 104021 (2008), 0806.3415

Adelberger et al.(2007)Adelberger, Heckel, Hoedl, Hoyle, Kapner, and Upadhye. E. G. Adelberger, B. R. Heckel, S. A. Hoedl, C. D. Hoyle, D. J. Kapner, and A. Upadhye, Phys. Rev. Lett. 98, 131104 (2007), hep-ph/0611223.

Mota and Shaw(2006). D. F. Mota and D. J. Shaw, Phys. Rev. Lett. 97, 151102 (2006), hep-ph/0606204.

Mota and Shaw(2007). D. F. Mota and D. J. Shaw, Phys. Rev. D75, 063501 (2007), hep-ph/0608078.

Upadhye(2012a). A. Upadhye, in 8th Patras Workshop on Axions, WIMPs and WISPs (AXION-WIMP 2012) Chicago, Illinois, July 18-22, 2012 (2012a), 1211.7066, URL http://inspirehep.net/record/1204935/files/arXiv:1211.7066.pdf.

Upadhye(2012b). A. Upadhye, Phys. Rev. D86, 102003 (2012b), 1209.0211.

Upadhye(2013). A. Upadhye, Phys. Rev. Lett. 110, 031301 (2013), 1210.7804

Lamoreaux and Buttler(2005). S. K. Lamoreaux and W. T. Buttler, Phys. Rev. E71, 036109 (2005).

Lambrecht and Reynaud(2011). A. Lambrecht and S. Reynaud, in Proceedings, 46th Rencontres de Moriond on Gravitational Waves and Experimental Gravity: La Thuile, Italy, March 20-27, 2011 (2011), pp. 199-206, 1106.3848, URL http://inspirehep. net/record/914261/files/arXiv:1106.3848.pdf

Brax et al.(2007a)Brax, van de Bruck, Davis, Mota, and Shaw. P. Brax, C. van de Bruck, A.-C. Davis, D. F. Mota, and D. J. Shaw, Phys. Rev. D76, 124034 (2007a), 0709.2075.

Brax and Davis(2015). P. Brax and A.-C. Davis, Phys. Rev. D91, 063503 (2015), 1412. 2080 .

Brax et al.(2010a)Brax, van de Bruck, Davis, Shaw, and Iannuzzi. P. Brax, C. van de Bruck, A. C. Davis, D. J. Shaw, and D. Iannuzzi, Phys. Rev. Lett. 104, 241101 (2010a), 1003.1605.

Almasi et al.(2015)Almasi, Brax, Iannuzzi, and Sedmik. A. Almasi, P. Brax, D. Iannuzzi, 
and R. I. P. Sedmik, Phys. Rev. D91, 102002 (2015), 1505.01763

Abele et al.(2010)Abele, Jenke, Leeb, and Schmiedmayer. H. Abele, T. Jenke, H. Leeb, and J. Schmiedmayer, Phys. Rev. D81, 065019 (2010), 0907.5447

Jenke et al.(2011)Jenke, Geltenbort, Lemmel, and Abele. T. Jenke, P. Geltenbort, H. Lemmel, and H. Abele, Nature Phys. 7, 468 (2011).

Jenke et al.(2014). T. Jenke et al., Phys. Rev. Lett. 112, 151105 (2014), 1404.4099

Nesvizhevsky et al.(2002). V. V. Nesvizhevsky et al., Nature 415, 297 (2002).

Westphal et al.(2007)Westphal, Abele, Baessler, Nesvizhevsky, Petukhov, Protasov, and Voronin.

A. Westphal, H. Abele, S. Baessler, V. V. Nesvizhevsky, A. K. Petukhov, K. V. Protasov, and A. Yu. Voronin, Eur. Phys. J. C51, 367 (2007), hep-ph/0602093

Brax and Burrage(2011). P. Brax and C. Burrage, Phys. Rev. D83, 035020 (2011), 1010. 5108

Jaeckel and Roy(2010). J. Jaeckel and S. Roy, Phys. Rev. D82, 125020 (2010), 1008.3536

Schwob et al.(1999)Schwob, Jozefowski, de Beauvoir, Hilico, Nez, Julien, Biraben, Acef, Zondy, and Clairon.

C. Schwob, L. Jozefowski, B. de Beauvoir, L. Hilico, F. Nez, L. Julien, F. Biraben,

O. Acef, J. J. Zondy, and A. Clairon, Phys. Rev. Lett. 82, 4960 (1999).

Simon et al.(1980)Simon, Schmitt, Borkowski, and Walther. G. G. Simon, C. Schmitt, F. Borkowski, and V. H. Walther, Nucl. Phys. A333, 381 (1980).

Brax et al.(2010b)Brax, van de Bruck, Davis, and Shaw. P. Brax, C. van de Bruck, A.-C. Davis, and D. Shaw, Phys. Rev. D82, 063519 (2010b), 1005.3735.

Damour et al.(2002)Damour, Piazza, and Veneziano. T. Damour, F. Piazza, and G. Veneziano, Phys. Rev. Lett. 89, 081601 (2002), gr-qc/0204094

Damour and Polyakov(1994). T. Damour and A. M. Polyakov, Nucl. Phys. B423, 532 (1994), hep-th/9401069

Williams et al.(2012)Williams, Turyshev, and Boggs. J. G. Williams, S. G. Turyshev, and D. Boggs, Class. Quant. Grav. 29, 184004 (2012), 1203.2150

Brax et al.(2012a)Brax, Davis, Li, and Winther. P. Brax, A.-C. Davis, B. Li, and H. A. Winther, Phys. Rev. D86, 044015 (2012a), 1203.4812.

Brax et al.(2012b)Brax, Davis, and Li. P. Brax, A.-C. Davis, and B. Li, Phys. Lett. B715, $38(2012 b), 1111.6613$

Brax et al.(2011a)Brax, Burrage, and Davis. P. Brax, C. Burrage, and A.-C. Davis, JCAP 1109, 20 (2011a), 1106.1573.

Bloomfield et al.(2015)Bloomfield, Burrage, and Davis. J. K. Bloomfield, C. Burrage, and A.-C. Davis, Phys. Rev. D91, 083510 (2015), 1408.4759.

Decca et al.(2007)Decca, Lopez, Fischbach, Klimchitskaya, Krause, and Mostepanenko. R. S. Decca, D. Lopez, E. Fischbach, G. L. Klimchitskaya, D. E. Krause, and V. M. Mostepanenko, Eur. Phys. J. C51, 963 (2007), 0706.3283.

Chen et al.(2016)Chen, Tham, Krause, Lopez, Fischbach, and Decca. Y. J. Chen, W. K. Tham, D. E. Krause, D. Lopez, E. Fischbach, and R. S. Decca, Phys. Rev. Lett. 116, $221102(2016), 1410.7267$

Bressi et al.(2002)Bressi, Carugno, Onofrio, and Ruoso. G. Bressi, G. Carugno, R. Onofrio, and G. Ruoso, Phys. Rev. Lett. 88, 041804 (2002), quant-ph/0203002.

Bressi et al.(2000)Bressi, Carugno, Galvani, Onofrio, and Ruoso. G. Bressi, G. Carugno, A. Galvani, R. Onofrio, and G. Ruoso, Class. Quant. Grav. 17, 2365 (2000).

Brax et al.(2011b)Brax, Burrage, Davis, Seery, and Weltman. P. Brax, C. Burrage, A.-C. Davis, D. Seery, and A. Weltman, Phys. Lett. B699, 5 (2011b), 1010.4536

Nitti and Piazza(2012). F. Nitti and F. Piazza, Phys. Rev. D86, 122002 (2012), 1202. 2105 .

Brax et al.(2007b)Brax, van de Bruck, Davis, Mota, and Shaw. P. Brax, C. van de Bruck, A.-C. Davis, D. F. Mota, and D. J. Shaw, Phys. Rev. D76, 085010 (2007b), 
30 Authors' Names

0707.2801

Zavattini et al.(2008). E. Zavattini et al. (PVLAS), Phys. Rev. D77, 032006 (2008), 0706. 3419.

Ehret et al.(2010). K. Ehret et al., Phys. Lett. B689, 149 (2010), 1004.1313

Steffen(2010). J. H. Steffen (GammeV-CHASE), PoS ICHEP2010, 446 (2010), 1011. 3802 .

Brax and Zioutas(2010). P. Brax and K. Zioutas, Phys. Rev. D82, 043007 (2010), 1004. 1846.

Brax et al.(2012c)Brax, Lindner, and Zioutas. P. Brax, A. Lindner, and K. Zioutas, Phys. Rev. D85, 043014 (2012c), 1110.2583.

Anastassopoulos et al.(2015). V. Anastassopoulos et al. (CAST), Phys. Lett. B749, 172 (2015), 1503.04561. 